\title{
Surgical and Transcatheter Pulmonary Valve Replacement: A Single Institution Comparison
}

Emily Barron; John W. Brown ${ }^{1,2}$; Mark W. Turrentine ${ }^{1,2}$, Mark Hoyer ${ }^{3}$; Mark H. Rodefeld ${ }^{1,2}$; Georges Ephrem ${ }^{4}$; Jeremy L. Herrmann ${ }^{1,2}$

${ }^{1}$ Indiana University School of Medicine, Indianapolis, IN, Division of Thoracic and Cardiovascular Surgery; ${ }^{2}$ Riley Children's Health, Indiana University Health, Indianapolis, IN;

${ }^{3}$ Division of Pediatric Cardiology, Department of Pediatrics, Riley Hospital for Children, Indiana University School of Medicine, Indianapolis, IN; ${ }^{4}$ Krannert Institute of Cardiology, Indiana University School of Medicine, Indianapolis, IN, United States

Background and Hypothesis: Pulmonary valve replacement (PVR) is one of the most commonly performed procedures for patients with congenital heart disease. Transcatheterbased PVR (TPVR) approaches have emerged as alternatives to surgical pulmonary valve replacement (SPVR), but few studies have directly compared clinical outcomes between the two interventions. Further characterization of performance between the two valve procedures may inform clinical decision-making.

Project Methods: Using institutional databases, we identified patients aged $\geq 9$ years who underwent either a TPVR or SPVR at Riley Hospital for Children between January 2009 and June 2020. Exclusions were made for previous endocarditis diagnosis, $<1$ year follow-up, and concomitant left heart procedures. Valve dysfunction was defined as $\geq$ moderate regurgitation or gradient $\geq 40 \mathrm{mmHg}$.

Results: 94 (TPVR, $\mathrm{n}=52$; SPVR, $\mathrm{n}=42$ ) patients met inclusion criteria. Average follow-up for SPVR and TPVR patients was 5.1(2.0,6.7) and 2.9(1.6,4.8) years, respectively $(p=0.007)$. The SPVR cohort was younger, had lower BMl, and underwent more prior sternotomies. Hospital length of stay was shorter after TPVR (1.0 day vs. 5.0 days, $p<0.001)$. Despite being younger, BSA-indexed valve size was larger in the SPVR cohort $\left(14.7 \mathrm{~mm} / \mathrm{m}^{2}\right.$ vs $\left.12.9 \mathrm{~mm} / \mathrm{m}^{2}, p<0.001\right)$. Short-term mortality ( $0 \%$ vs $2 \%, p=0.36)$, endocarditis ( $0 \%$ vs $6 \%, p=0.11)$, and reintervention $(12 \%$ vs $8 \%, p=0.49)$ did not differ between groups. Intermediate-term valve dysfunction/failure was greater in SPVR patients $(29 \%$ vs $12 \%, p=0.04)$ with time to dysfunction $809(421,1565)$ and 1184 $(181,1627)$ days for SPVR and TPVR, respectively. Valve implantation failure due to pre-stent migration occurred in $4 \%$ of TPVR cases; one required surgical intervention.

Conclusion and Potential Impact: In patients undergoing PVR at our institution, rates of mortality and infective endocarditis are similar between interventions. Intermediate-term valve dysfunction/failure was greater in SPVR cohort, but length of follow-up was significantly longer in these patients. Reintervention rates were similar between procedures.

Word Count: 296/300 